\title{
LA VIOLENCIA SEXUAL DESDE LA PERSPECTIVA DE GÉNERO. APUNTES PARA UNA REFORMA PENAL EN CUBA
}

\author{
Lisett D. Páez Cuba ${ }^{1}$ \\ Jorge Luis Silva González ${ }^{2}$ \\ Laidiana Torres Rodríguez ${ }^{3}$
}

\begin{abstract}
The updating of the economic model and the legal system in Cuba, after the recent approval of the Constitution of the Republic of 2019, requires the consequent insertion of a gender perspective in the legal spaces where the actors of the Law play a significant role. In this regard, this study aims to assess the legal regulation of the types of sexual violence provided for in the Cuban Criminal Code, as guidelines for legal reform based on an adequate gender perspective. For this, scientific methods such as theoretical-legal, exegetic-analytical, legal comparison and documentary review were used. Its use allowed to identify two essential problems: the absence of emerging figures of social practice (feminicide, homophobia, transphobia and cyberbullying), and the insufficient and discriminatory regulation of crimes already foreseen (rape, pedophilia with violence, lascivious abuse and trafficking in persons). A legal comparison was also made of the criminal offenses regulated in Spain, Argentina, Bolivia, Venezuela and Peru, whose references allowed visualizing the challenges and perspectives in the context of legislative reform in which Cuba is immersed, for a correct configuration of the gender approach in the Cuban legal system.
\end{abstract}

1 Licenciada en Derecho. Doctora en Ciencias Pedagógicas. Profesora Auxiliar del Departamento de Derecho y Vicedecana de Investigación y Posgrado de la Facultad de Ciencias Sociales y Humanidades de la Universidad de Pinar del Río, Cuba. ORCID: 0000-0001-89077562. lisett@upr.edu.cu

${ }^{2}$ Licenciado en Derecho. Máster en Desarrollo Social. Profesor Asistente del Departamento de Derecho de la Universidad de Pinar del Río, Cuba. Estudiante de la Maestría en Derecho Constitucional y Administrativo de la Facultad de Derecho de la Universidad de La Habana. ORCID: 0000-0002-0214-9744. silva@upr.edu.cu

${ }^{3}$ Licenciada en Derecho. Profesora Instructora del Departamento de Derecho de la Facultad de Ciencias Sociales y Humanidades de la Universidad de Pinar del Río, Cuba. Estudiante de la Maestría en Derecho Constitucional y Administrativo de la Facultad de Derecho de la Universidad de La Habana. ORCID:_0000-0003-0534-9247. laidiana.torres@upr.edu.cu 
Keywords: gender; Cuban Criminal Code; criminal reform; sexual violence

\section{Introducción}

Las relaciones inequitativas de género trascienden de manera particular al escenario socio-jurídico, donde se tipifican determinadas conductas con la imposición de sanciones. En la actualidad, el tratamiento de la temática de género -desde la arista jurídica- continúa siendo un imperativo para las sociedades contemporáneas, teniendo en cuenta que dentro de los Objetivos de la Agenda 2030 para el Desarrollo Sostenible se localiza en el numeral quinto la igualdad de género, aspiración a la que tributan las Ciencias Jurídicas desde los contextos de reformas legales.

Si bien las realidades latinoamericanas difieren de otras más severas como el caso de Arabia Saudita, donde la mujer es discriminada, aún queda mucho camino por recorrer si de equidad de género se trata, constituyendo una necesidad que los actores sociales que responden a políticas públicas tengan un rol más activo y eficaz.

En correspondencia con ello, el análisis de la problemática de género -como categoría transversal a los procesos con repercusión jurídica y trascendencia social- en materia de investigaciones gana mayor presencia cada año en la agenda de talleres y eventos científicos de Derecho, por hacer prevaler el Principio de Igualdad plasmado en la recientemente promulgada Constitución de la República de Cuba el 10 de abril de 2019 y que hace referencia a que todos los ciudadanos gozan de iguales derechos y están sujetos a iguales deberes; asimismo, que la mujer y el hombre gozan de iguales derechos en lo económico, político, cultural, social y familiar.

Si bien los preceptos de la Constitución, evidencian como regla general de que en una sociedad socialista ambos sexos tienen igualdad ante la Ley, algunas normas del ordenamiento jurídico y específicamente del Derecho Penal, son tendentes a reproducir patrones discriminatorios que, en ocasiones ubican en posición de desventaja a las mujeres al sustentarse en políticas igualitarias, mientras que en otras, se conciben tratamientos diferenciados, cuando lo más conveniente sería una formulación única para ambos sexos.

Las mujeres jóvenes y niñas son especialmente vulnerables por las diferentes tipologías de violencia sexual que están previstas en el Código Penal cubano, las que 
tienen un carácter arbitrario en su configuración, pues penalizan con mayor severidad los supuestos donde el hombre aparece como sujeto pasivo.

En tal sentido, el presente estudio tiene como objetivo valorar la regulación jurídica de las tipologías de violencia sexual previstas en el Código Penal cubano, de manera que se fundamenten pautas para una consecuente y necesaria reforma de esta ley sustantiva. Para ello se emplearon métodos científicos tales como el teórico-jurídico, el exegético-analítico, la revisión documental y el de comparación jurídica. En el caso de este último, las deficiencias identificadas en la realidad socio-jurídica cubana dieron paso a establecer una comparación de los ilícitos penales regulados en España, Argentina, Bolivia, Venezuela y Perú, los cuales resultan referentes para el contexto latinoamericano y caribeño, y en particular para Cuba. Su sistematización implica contextualizar la equidad en toda su configuración teórica, con especial impacto en la identificación de otras formas de violencia sexual no visualizadas a priori en la Isla.

La investigación grosso modo ofrece un análisis crítico que enaltece la representación social de las mujeres jóvenes y niñas desde el Derecho, con particular atención a las propuestas de modificación de la ley penal sustantiva. Se visualizan por tanto los retos y perspectivas en el contexto de la reforma legislativa en que Cuba está inmersa, para una adecuada configuración del enfoque de género en todo el ordenamiento jurídico cubano.

Es preciso acotar, que el presente estudio en la materia de Derecho Penal, da continuidad a la investigación desarrollada con el título "Fundamentos para la prevención de desigualdades desde el sector jurídico cubano en función del desarrollo social”, publicado por el este propio Periódico del Núcleo de Estudios y Pesquisas sobre Género y Direito en el volumen siete, número dos del año 2018.

\section{Referentes teóricos sobre el enfoque de género desde la perspectiva de la equidad}

La palabra género designa a una categoría gramatical y no es, por tanto, sinónimo de sexo. Pero también es cierto que el género es, como el sexo, una característica que comparten hombres y mujeres. Se trata de tipologías completamente diferentes: el sexo es un rasgo fisiológico, y el género, una categoría gramatical que solo afecta indirectamente a hombres y mujeres a través de la palabra que los designa. 
Desde la teoría de género varios han sido los autores y las autoras que se han referido a lo que debe entenderse por género desde finales del siglo pasado. Para Lagarde (1996), por ejemplo, es una categoría que abarca lo bio-socio-sico-econopolítico-cultural, que analiza la síntesis histórica que se da entre todas estas esferas y por tanto, su construcción es nacida de un histórico proceso de condicionamientos que atribuye rasgos, características y potencialidades a cada sexo, y que más que basarse en diferencias biológicas: es un patrón cultural.

Según Caram (1996) y coincidiendo con Lagarde, es un proceso histórico de condicionamiento social y cultural que atribuye rasgos, características y potencialidades a los sexos, conformando una forma de ser y actuar, y que aunque se basa en las diferencias biológicas es un patrón cultural.

Coincide Fleitas (2000), en que el género es un concepto que se refiere a una construcción social y cultural, que se ha incidido en la formación de una identidad femenina subordinada y que tradicionalmente ha sido enfrentada al sexo como indicativo de procesos biológicos que significan el ser mujer frente al ser hombre, los cuales atendiendo a su carácter natural, no determinan diferencias de posición social.

Sin embargo, Proveyer (2005) lo define con una visión más amplia, plantea que es un conjunto de prácticas, símbolos, representaciones, normas y valores sociales que las sociedades elaboran a partir de la diferencia sexual anátomo-fisiológica y que dan sentido a las relaciones entre las personas sexuadas, alude a mujeres y hombres y a las relaciones que entre ellos se producen. Las cuestiones relativas al género tienen, por tanto, un contenido relacional; son social y culturalmente e involucran a ambos géneros.

Las definiciones expuestas con anterioridad evidencian que existe consenso de criterios en torno a la categoría desde el punto de vista doctrinal y se entenderá a los efectos del presente estudio como aquel "proceso de construcción social y/o cultural que asigna a cada persona según su sexo -desde el momento de su nacimiento-, características, roles, valores y normas de todo tipo, ya sean sociales, políticas, económicas, jurídicas, culturales y psicológicas" (Silva González, Pérez Véliz y Delgado Rodríguez, 2017, p. 1495).

Asimismo, se concibe que el tratamiento de género en función de una sociedad más justa, debe valorarse teniendo en cuenta el principio de equidad como principio 
general del Derecho y a partir de la concepción romana de equidad de dar a cada cual lo que le toca en el tratamiento y aplicación del Derecho.

En 1996, Marcela Lagarde refirió que el enfoque de género es sinónimo de perspectiva de género y contiene también el análisis de género, permitiendo analizar y comprender las características que definen a las mujeres y a los hombres de manera específica, así como sus semejanzas y diferencias.

Desde el punto de vista del Derecho, Staff (1999), plantea que la perspectiva de género, significa la promoción de la igualdad y de las nuevas identidades de género, reduciendo o eliminando las causas y efectos de la discriminación en el ámbito jurídico.

A partir de los criterios de las autoras anteriores se considera que la equidad de género en el campo del Derecho y en otros ámbitos, no supone que hombres y mujeres sean iguales, pero sí que tengan acceso y disfruten de los bienes sociales, las oportunidades y los recursos sin distinción alguna.

El énfasis en la equidad o en el empoderamiento de género, no presupone un modelo definitivo de igualdad para todas las sociedades, pero refleja la preocupación de que ambos sexos, aunque no sean iguales por razones biológicas, sí tengan las mismas facilidades para tomar decisiones y trabajen juntos para lograrlo en virtud del Principio de Igualdad reflejado en la Constitución cubana de 2019 y que debe hacerse extensivo a todo el ordenamiento jurídico, en especial a la rama objeto de investigación: el Derecho Penal.

Valoraciones sobre el enfoque de género en el Código Penal cubano, como base para el estudio comparativo y la fundamentación de los retos actuales

La norma sustantiva cubana, en el caso del Código Penal o Ley No. 62 de 29 de diciembre de 1987, puesto en vigor el 30 de abril de 1988 y modificado por el Decreto-Ley No. 150 de junio de 1994, con sus dos últimas modificaciones: el DecretoLey 175, de 17 de junio de 1997 y la Ley 87 de 16 de febrero de 1999, regula en el capítulo XI los delitos contra el normal desarrollo de las relaciones sexuales.

Las figuras básicas de los delitos de Violación, Pederastia con Violencia y Abusos lascivos respectivamente, plantean: 
Artículo 298.1: Se sanciona con privación de libertad de cuatro a diez años al que tenga acceso carnal con una mujer sea por vía normal o contra natura, siempre que en el hecho concurra alguna de las circunstancias siguientes:

a) usar el culpable de fuerza o intimidación suficiente para conseguir su propósito;

b) hallarse la víctima en estado de enajenación mental...o carente de la facultad de dirigir su conducta.

Artículo 299.1: El que cometa actos de pederastia activa empleando violencia o intimidación, o aprovechando que la víctima esté privada de razón o de sentido o incapacitada para resistir, es sancionado con privación de libertad de siete a quince años.

Artículo 300.1: El que, sin ánimo de acceso carnal, abuse lascivamente de una persona de uno u otro sexo, concurriendo cualquiera de las circunstancias previstas en el apartado 1 del artículo 298, incurre en sanción de privación de libertad de seis meses a dos años o multa de doscientas a quinientas cuotas.

Con la regulación de dichos delitos en el Código Penal vigente desde 1987, según Rega (2003), se incorporan actos, agresiones y abusos sexuales que atentan directa o indirectamente contra el normal desenvolvimiento de las relaciones sexuales. Incluye tanto la libertad del sujeto de elegir de forma autónoma en el ámbito de la sexualidad lo referente a la excitación y satisfacción sin traspasar las barreras del Derecho Penal, así como de elegir su pareja, determinar la opción sexual que se prefiera en cada momento.

El enfoque de género a partir de un análisis crítico en ambos delitos y desde la equidad, atendiendo a los sujetos que intervienen y el marco sancionador, se puede apreciar que el legislador cubano formuló dos figuras de delitos analógicamente semejantes por presentar conductas similares, pero con diferencias para ambos sexos.

En el caso de la Violación, según la disposición del artículo, se presenta sólo a la mujer como sujeto pasivo del delito, mientras que en la Pederastia con violencia la acción recae sobre el hombre.

En el caso de la sanción, se evidencia en el marco sancionador del delito de violación de cuatro a diez años, a la mujer, con un tratamiento desventajoso con respecto al marco sancionador del hombre en la pederastia con violencia, lo que evidencia o sugiere que el legislador otorgó con la plasmación de tal diferenciación, una mayor protección a la integridad sexual del hombre de siete a quince años, como si fuera de superior valor a la integridad sexual de la mujer de cuatro a diez años. 
Según la consideración de los autores, estamos en presencia de una concepción expresada en Ley, de carácter tradicionalista y sexista, que no otorga el mismo tratamiento para ambos sexos, aun cuando la propia práctica jurídica y social indica que la Violación tiene mayor radicación que la Pederastia con violencia.

Al decir de Rega, "el término violación sin dudas de gran tradición cultural, criminológica y legal, no ha logrado romper la idea que la asocia con la relación heterosexual, donde solo puede ser sujeto pasivo la mujer" (2003, p.137).

Se está en definitiva, en presencia de disposiciones normativas que suponen una diferencia de género por ende discriminatoria, que reconoce que en materia penal en Cuba, no se ha logrado superar el tratamiento del contexto del delito de violación, con cualquier persona, dígase hombre o mujer como sujeto pasivo del delito.

Es preciso aclarar -partiendo del análisis del enfoque de género-, que no ocurre lo mismo en el delito de abusos lascivos, donde sí se contemplan como sujetos pasivos a ambos sexos, concurriendo inclusive cualquiera de las circunstancias previstas en el apartado 1 del artículo 298 Violación, lo que presupone y ratifica la concepción tradicionalista predominante en el modo de pensamiento del legislador cubano al plasmar las figuras delictivas de la violación y la pederastia con violencia.

Por ende, al realizar un análisis comparativo de los delitos de Violación y Pederastia con Violencia desde la perspectiva de la equidad, se identifica un elemento sumamente discriminatorio en torno al marco penal, toda vez que la sanción de privación de libertad es más severa para los supuestos de violación sexual a hombres (7 a 15 años) que para la violación cometida hacia mujeres (4 a 10 años). De igual forma, se prevé un marco penal de 7 a 15 años para las adolescentes víctimas que tienen entre 12 y 14 años; sin embargo, se ofrece mayor protección a la integridad sexual del adolescente varón menor de 14 años como víctima, pues el marco penal en este caso resulta de 15 a 30 años o muerte.

$\mathrm{Al}$ ofrecer mayor connotación a la violación cometida de un hombre hacia otro, que aquella consumada de un hombre hacia una mujer, el enfoque de género es de carácter tradicionalista y sexista. Incluso, quedan fuera de la ley penal los supuestos de violación de mujer a mujer o aquellos cometidos contra personas transexuales. Asimismo existen tipicidades específicas que no encuentran asidero en la ley cubana, 
tales como las asociadas a la penetración de objetos y la felatio in ore, supuestos estos que configurarían el delito de Abusos Lascivos. De tal manera, existe una perspectiva reduccionista del tipo penal de Violación, al configurarse solo cuando se realiza la penetración de miembro viril en cavidad con esfínter.

Se considera que dicha problemática hubiera sido resuelta con una única norma que contemplara a ambos sexos como sujetos pasivos de los dos delitos antes mencionados y como se evidencia en materia penal en países del Sistema de Derecho Romano-Francés como Venezuela, Argentina, España y Bolivia (Silva \& Pérez, 2018).

Estudios comparados de la violencia sexual. Referentes desde las legislaciones de Venezuela, Argentina, España, Bolivia y Perú

Las deficiencias identificadas en la realidad socio-jurídica cubana dieron paso a establecer una comparación jurídica de los ilícitos penales regulados en España, Argentina, Bolivia, Venezuela y Perú, los cuales resultan referentes para el contexto latinoamericano y caribeño, y en particular para Cuba. Su sistematización implica contextualizar el principio de equidad en toda su configuración teórica, con especial impacto en la identificación de otras formas de violencia sexual no visualizadas a priori en la Isla.

En materia de Derecho Comparado, en la actualidad, una mirada al enfoque de género bajo la égida de la equidad, específicamente en el delito de violación en materia penal, sugiere revisar el tratamiento dado por el Código Penal de países específicos. España, porque es el tronco del cual emanó todo el ordenamiento jurídico latinoamericano; Venezuela y Bolivia porque son de los casos cuyo enfoque sociopolítico es más semejante al cubano, y Argentina porque es uno de los países que se reconoce en América Latina como de mayor desarrollo de su ordenamiento jurídico y su actividad jurisprudencial.

El Código Penal de Venezuela, divulgado por la Gaceta Oficial N 5.494 Extraordinario del 20 de octubre de 2000, plantea en su Título VIII, Capítulo I "De la violación, de la seducción, de la prostitución o corrupción de menores y de los ultrajes al pudor", artículo 375, que "El que por medio de violencias o amenazas haya 
constreñido a alguna persona, del uno o del otro sexo, a un acto carnal, será castigado con presidio de cinco a diez años".

Por su parte, el Código Penal de Argentina actualizado o Ley 11.179 de 1984, en su Capítulo II, artículo 119 plantea:

Será reprimido con reclusión o prisión de seis meses a cuatro años el que abusare sexualmente de persona de uno u otro sexo cuando, ésta fuera menor de trece años o cuando mediare violencia, amenaza, abuso coactivo o intimidatorio de una relación de dependencia, de autoridad, o de poder, o aprovechándose de que la víctima por cualquier causa no haya podido consentir libremente la acción.

El Código Penal de España de 1995, en el artículo 179, también asume el concepto amplio de violación, que asimila no solo como sujeto pasivo a la mujer sino también al hombre; este plantea que:

Cuando la agresión sexual consista en acceso carnal por vía vaginal, anal o bucal, o introducción de objetos por cualquiera de las dos primeras vías, el responsable será castigado como reo de violación, a prisión de seis a doce años (Rega, 2003, p.135).

Por último el Código Penal de Bolivia, modificado por la Ley 2033 de 1999 en su artículo 308 dice: "Quien empleando violencia física o intimidación, tuviera acceso carnal con persona de uno u otro sexo; penetración anal o vaginal o introdujera objetos con fines libidinosos (...)”.

Una formulación de referencia aparece en el Código Penal peruano, Capítulo IX: Violación de la libertad sexual, Artículo 170: "El que con violencia o grave amenaza, obliga a una persona a tener acceso carnal por vía vaginal, anal o bucal o realiza otros actos análogos introduciendo objetos, o partes del cuerpo por algunas de las dos primeras vías, será reprimido con (...)”.

$\mathrm{Al}$ analizar cada uno de los casos citados anteriormente se llega a la conclusión de que existen diferencias entre las figuras delictivas de la violación de los países expuestos y la norma cubana que contempla dicho delito en el Código Penal.

La diferencia más significativa radica en los sujetos pasivos en los que recae la Violación, pues tanto España como Venezuela, Bolivia, Argentina y Perú, lo regulan en una misma normativa para ambos sexos, sin necesidad de establecer la misma conducta con marcos sancionadores diferentes para el hombre o la mujer, otorgándole a ambos el mismo valor e importancia. Sin lugar a dudas, estos constituyen modelos de países 
cuyos legisladores han tenido en cuenta la equidad de género respecto al delito de Violación, un patrón a seguir la futura reforma penal en Cuba.

\section{Retos del enfoque de género en el Derecho Penal cubano en torno a la violencia sexual}

Las pautas para el perfeccionamiento de la ley penal cubana en materia de género van desde considerar la inexistencia de una figura de Feminicidio hasta la propia denominación de la figura penal asociada a la transgresión de la libertad sexual. En tanto en el Código Penal cubano se emplea el término genérico "Violación", el que pudiera aludir a Violación de domicilio, Violación de la correspondencia, entre otros; se evidencia una ambigüedad, cuando en realidad debería denominarse al delito como "Violación de la libertad sexual", de manera que quede explícito el bien jurídico protegido.

Asimismo existen tipicidades específicas que no encuentran asidero en la ley cubana, pues si bien la norma penal debe ser general y abstracta, no se prevén actualmente como Violación las conductas asociadas a la penetración de objetos y el sexo oral, lo que sí se establece en otras legislaciones de Derecho Comparado. Esta situación conlleva a un aparente concurso de normas penales entre las figuras de Violación y Abusos Lascivos, siendo esta última la aplicable cuando se realizaron prácticas lascivas sin consumarse la penetración de miembro viril en la cavidad con esfínter.

Un elemento sumamente discriminatorio en torno a la equidad de género resulta ser el marco penal diferenciador para las figuras de Violación y Pederastia con Violencia. Para este último supuesto la figura básica es más severa, sancionando por ende con mayor connotación la violación cometida de un hombre hacia otro, que aquella que consuma un hombre hacia una mujer.

Otro de los supuestos que pudieran tener lugar en la cotidianidad es el de la Violación dentro del matrimonio, cuestión profusamente discutida en la doctrina. Realmente existen los elementos de tipicidad para configurar el delito, mas no se identifica la situación como tal, al preponderar "supuestamente" los derechos del cónyuge sobre la libertad sexual de su esposa. Realmente la situación trasciende las 
barreras del Derecho Penal, pues aun estando regulado en ley, las mujeres víctimas no lo identifican como delito y en otras ocasiones no establecen la denuncia. Este último supuesto limita la perseguibilidad de oficio del delito de Violación, que por su carácter se semipúblico requiere de la denuncia del perjudicado, según estipula el artículo 309 del Código penal vigente.

Por ende, las propuestas de perfeccionamiento estarían dirigidas hacia la tipificación de una figura única para ambos sexos, sin diferenciar en Violación o Pederastia, sino definiendo sujetos generales, tal como lo hacen disímiles Códigos penales analizados desde el Derecho Comparado. De tal manera, los principales retos que encuentra una adecuada perspectiva de género en el Derecho Penal cubano van dirigidos a su reconfiguración normativa.

De cara a una reforma penal en Cuba, las perspectivas de un coherente enfoque de género en la ley penal sustantiva implican, respecto al delito de Violación, los siguientes elementos:

1. Adecuada denominación de la conducta, alusiva a la violación "de la libertad sexual".

2. Inclusión de otras tipicidades referidas al sexo oral y la penetración de objetos.

3. Igualdad de sanción penal para los supuestos de violación sexual a hombres o mujeres.

4. Unicidad de figura jurídica para la tipificación de conductas de sujeto general, tanto activo como pasivo.

A tono con una adecuada perspectiva de género en el Código Penal, se valora además la figura de la Trata de Personas. Esta última evidencia un carácter restrictivo en su formulación, referida solo a la explotación sexual, excluyendo otras tipologías como los matrimonios forzados, la adopción irregular, la extracción de órganos, la esclavitud reproductiva y otras formas modernas de esclavitud. Por ende, la mera delimitación a la violencia sexual resulta insuficiente y amerita de una nueva formulación que incluya la violencia psicológica, material, doméstica, intrafamiliar y otras que convierten a mujeres y niñas en sujetos con especial necesidad de protección. 
Otras formas de violencia sexual que también requieren de un análisis crítico resultan aquellas como el feminicidio, la homofobia, la transfobia y el ciberacoso. Ninguna de ellas encuentra respaldo en la actualidad jurídica cubana. Así aparecen muchas veces las amenazas y coacciones que devienen posteriormente en un Feminicidio, delito que no está tipificado explícitamente en la ley penal. De igual forma, el acoso sexual del cual son víctimas las mujeres, incluso adolescentes cibernautas, a través de las redes sociales, es una conducta que queda impune en la generalidad de los casos. Estas constituyen tipologías de violencia sexual de índole psicológica, las que, sin constituir agresión física, afectan la integridad sexual y moral de las víctimas, su derecho a la imagen, al honor y al respeto de la identidad sexual.

Este análisis crítico de la ley penal cubana enaltece la representación social de las mujeres jóvenes y niñas desde el Derecho, con particular atención a las propuestas de modificación de la ley sustantiva. Se visualizan por tanto los retos y perspectivas en el contexto de la reforma legislativa en que Cuba está inmersa, para una adecuada configuración del enfoque de género en todo el ordenamiento jurídico cubano.

Grosso modo los principales aspectos propuestos para una reforma penal en Cuba, en pos de una adecuada perspectiva de género, implican la reconfiguración de un tipo penal genérico que proteja indistintamente la libertad sexual del hombre y la mujer, en sus diversas manifestaciones y tipologías, de manera equitativa tanto en la configuración de las figuras de delitos como en la cuantía de sus sanciones penales. De igual forma se propone la modificación de la figura de Trata de Personas y la inclusión de nuevas tipologías de violencia de género que potencien valores de justicia y seguridad jurídica. Su impacto social irradia a la población juvenil, en tanto las contribuciones teóricas de la presente investigación resultan, a su vez, referentes jurídicos insoslayables para la implementación de la Campaña Nacional por la no violencia hacia mujeres y niñas.

\section{Conclusiones}

El enfoque de género en el Código Penal cubano en los artículos 298.1 (Violación) y 299.1 (Pederastia con Violencia) correspondientes a la Sección Primera del Capítulo I "Delitos contra el normal desarrollo de las relaciones sexuales", del 
Título XI, del Código Penal, es de carácter tradicionalista y sexista en tanto ofrece mayor protección a la integridad sexual del hombre que a la de la mujer, lo que no se ajusta a la equidad sustentada en el Principio constitucional de Igualdad. En tal sentido, los principales aspectos a tener en cuenta para una reforma penal en Cuba, en pos de una adecuada perspectiva de género, implican la reconfiguración de un tipo penal genérico que proteja indistintamente la libertad sexual del hombre y la mujer, en sus diversas manifestaciones y tipologías, de manera equitativa, tanto en la configuración de las figuras de delitos como en la cuantía de sus sanciones penales.

Por otra parte, la delimitación a la violencia sexual resulta insuficiente y merece una nueva formulación que incluya diversos tipos de violencia como la psicológica, la material, la doméstica, la intrafamiliar y otras que convierten a mujeres y niñas en sujetos con especial necesidad de protección, tales como el feminicidio, la homofobia, la transfobia y el ciberacoso, las cuales tienen presencia en países del contexto latinoamericano y caribeño como España, Argentina, Bolivia, Venezuela y Perú.

\section{Referencias bibliográficas}

Bolivia. Código Penal, Ley no 1768 modificado por la Ley 2033/ 1999. Citado 15 jul 2017. Disponible en http://www2.congreso.gob.pe/sicr/cendocbib/con4_uibd.nsf/46CA85EA04EBEE40052 57D2C007035D4/\$FILE/CODIGOPENALDEBOLIVIA.pdf

Caram, T. (1996). La Mujer cubana y la participación social: educación y ciencia. Tesis de Maestría, Programa FLACSO Cuba, Universidad de La Habana.

Cuba. Ley No. 62. Código Penal de Cuba de 1987.Publicada en Colección Jurídica. Ministerio de Justicia. La Habana, Cuba. Código Penal de Argentina, 1984.

España. Código Penal. Ley Orgánica 10 (1995, 23 de noviembre). BOE no. 281/24 de noviembre de 1995. España: Editorial QUILES. Disponible en https://vlex.es/tags/codigo-penal-españa-730039

Fleitas, R. (2000): “Identidad femenina y maternidad adolescente", Tesis de doctorado, Departamento de Sociología, Universidad de La Habana, Cuba. 
Lagarde, M. (1996) "El género”, fragmento literal: 'La perspectiva de género', en Género y feminismo. Desarrollo humano y democracia, España: Ed. horas y HORAS.

Perú. Decreto Legislativo No. 635. Código Penal. Edición Especial, abril de 2018. Lima: Jurista Editores.

Proveyer, C. (2005). Selección de lecturas de Sociología y Política Social de Género. La Habana: Editorial Félix Varela, 2005: 1. En Gomáriz, Enrique. Los estudios de género y sus fuentes epistemológicas: periodización y perspectivas. Chile. Editorial FLACSO, 1922.

Rega, E. (2003): Derecho Penal Especial, tomo II. La Habana: Ed. Félix Varela.

Silva-González, J.L., Pérez-Véliz, A. \& Delgado-Rodríguez, Y. (2017). Analizando la Masculinidad en la Ley General de la Vivienda Cubana para la Formación Profesional de Juristas. Multidisciplinary Journal of Gender Studies, 7(1), 1486-1505. doi: 10.17583/generos.2018.3132

Silva, J.L., y Pérez, A. (2018). Fundamentos para la prevención de desigualdades desde el sector jurídico cubano en función del desarrollo social. Revista Gênero e Direito. Periódico do Núcleo de Estudos e Pesquisas sobre Gênero e Direito. V. 7 - № 02, pp. 113-140. Disponible en https://periodicos.ufpb.br/ojs2/index.php/ged/index

Staff, M, (2000). Género y Derecho. Curso de Formación en Género. Módulo 3. Instituto de la Mujer de la Universidad de Panamá. Editora Sibauste, Primera edición. Legalinfo, Panamá. Disponible en http://www.legalinfopanama.com/articulos/articulos_21f.htm

Venezuela. Código Penal (2000, 20 de octubre). Gaceta Oficial de la República Bolivariana de Venezuela $\mathrm{N}^{\circ} 5.494$ (Extraordinario) 2000. Citado 8 dic 2016. Disponible en http://www.oas.org/juridico/spanish/mesicic3_ven_anexo6.pdf 